\title{
Characteristic of the spinal muscular atrophy cell model
}

\author{
Ovechkina V. ${ }^{1,2 *}$, Valetdinova K. ${ }^{1,2}$ \\ ${ }^{1}$ Novosibirsk State University, Novosibirsk, Russia \\ ${ }^{2}$ Institute of Chemical Biology and Fundamental Medicine, SB RAS, Novosibirsk, Russia \\ *e-mail:Vs_ovechkina@mail.ru
}

Key words: spinal muscular atrophy, induced pluripotent stem cells, cell model

Motivation and Aim: After the induced pluripotent stem cells were obtained by the Yamanako group, experiments on the development of cellular models of disease have become widespread in medical biology. Here we performed a comparative description of a previously obtained cellular model of spinal muscular atrophy (SMA) at different stages of differentiation. We compared the expression of well-known and newly founded by genome-wide linkage analysis genes, involved in molecular pathogenesis of SMA. Methods and Algorithms: We utilized induced pluripotent stem cells, obtained from conditionally healthy patient and patients with SMA I and II types [1]. Using the open Internet resource BLAST we selected primer pairs to estimate by Real-time PCR the number of SMN1, SMN2, PLS3, SLC23A2, NCALD, RPL6, CDKA2P1 genes [2-4], which products are involved in the development of SMA. Statistical processing of the results were carried out using the non-parametric Kruskal-Wallis criterion for independent groups using the Statistica 64. Differences were considered significant when the probability parameter value was $p<0.05$.

Results: We shown a decrease in the expression level of the full-sized SMN transcript at all stages of neural differentiation in cell models with the SMA phenotype of types I and II in comparison with control. An increase in the level of the incomplete $S M N$ transcript was also detected. We shown a change in the transcription of modifier genes: in case of SMA type I, the number of RPL6 transcripts increases in mature motor neurons, and the expression level of PLS3, NCLD, CDK2AP1 decreases.

Conclusion: The earlier obtained cell model of SMA complies well-known literary characteristics of mature motor neurons with SMA phenotype and can be used for further molecular applications. Newly founded by genome-wide linkage analysis genes (RPL6 and $C D K 2 A P 1)$ can be also involved in pathogenesis of SMA.

Acknowledgements: Supported by RSF (17-75-10041).

\section{References}

1. Valetdinova K.R. Spinal muscular atrophy model system based on induced pluripotent stem cells: Abstract dis. ... Ph.D. in Biology: 03.02.07. Novosibirsk, 2016 (in Russian).

2. Alrafiah A., Karyka E., Coldicott I., Iremonger K., Lewis K.E., Ning K., Azzouz M. Plastin 3 promotes motor neuron axonal growth and extends survival in a mouse model of spinal muscular atrophy. Mol. Ther. Methods Clin. Dev. 2018;31(9):81-89.

3. Zheleznyakova G.Y., Nilsson E.K., Kiselev A.V., Maretina M.A., Tishchenko L.I., Fredriksson R., ... Schiöth H.B. Methylation levels of SLC23A2 and NCOR2 genes correlate with spinal muscular atrophy severity. PLOS ONE. 2015;10(3):1-14.

4. Riessland M., Kaczmarek A., Schneider S., Swoboda K.J., Löhr H., Bradler C., ... Wirth B. Neurocalcin delta suppression protects against spinal muscular atrophy in humans and across species by restoring impaired endocytosis. American J. Human Gen. 2017;100(2):297-315. 